\title{
Characteristics of some anatomical landmarks on the anterior face of the maxilla
}

Discipline of anatomy, Department I - preclinical disciplines, Faculty of medicine, University "Ovidius" Constanţa

\begin{abstract}
The canine bossa (not listed in TA) was clearly visible in $80 \%$ of cases, less prominent in $12 \%$ of cases or even absent in $8 \%$ of cases. The most commonly is oval in shape with the long axis oriented vertically or slightly oblique infero-medially. In $60 \%$ of cases the two fossae showed approximately equal sizes. For the rest of the $40 \%$ of the cases, the left one was larger ( 24 cases) than the right one. In $12 \%$ of cases we found a dehiscent anterior wall of the canine fossa for more than half of its height. The myrtiform fossa (also not mentioned in the TA) was oval, with the long axis oriented vertically and with same dimensions bilaterally in $60 \%$ of cases. In $2 / 3$ of cases was evident (deeper) on the right. In $28 \%$ of cases we found it rounded, more frequently also on the right and in $12 \%$ of cases we found it as having an irregular shape. In about $10 \%$ of cases have it was almost flat. The canine fossa (Fossa canina) has various shapes, more frequently rounded with an average diameter of $0.8-0.9 \mathrm{~cm}$. In $30 \%$ of cases was oval, with vertical long axis of $1 \mathrm{~cm}$ in and $0.7 \mathrm{~cm}$ width. The cases of rounded canine fossa, well circumscribed and deeper, were more prevalent on the left. The infraorbital foramen (Foramen infraorbitale) was circular in equal proportions (in half of the cases), with a diameter of 2.5-5 mm, or oval, with $3-5 \mathrm{~mm}$ vertical axis and $1.5-3 \mathrm{~mm}$ horizontal axis. Note the difference in shape in the same skull: circular on the left and oval on the right (most frequent case) or vice versa. The distance from the infraorbital border is variable, being lower on the left in $65 \%$ of cases. Compared to the piriform aperture, the infraorbital foramen is located at 0.9 to $1.7 \mathrm{~cm}$ on the right and at $1.2-1.7 \mathrm{~cm}$ on the left. We encountered five cases with double infraorbital foramen. The intermaxillary suture is vertical in only $35 \%$ of cases. In other cases it is deviated to the left in $40 \%$ of cases, and in $25 \%$ of the Iliescu D.M.
\end{abstract}

Department of Anatomy, Faculty of medicine, University "Ovidius" Constanța, Romania

Aleea Universitatii, Nr. 1, Campus B

Constanţa, Romania

dan@anatomie.ro cases is oriented to the right. The degree of inclination is variable, making with the horizontal an angle between 95 to 1100 . The alveolar process (Procesus alveolaris) is wider posteriorly. There is a difference in width rightto-left, being wider on the right side in $40 \%$ of cases $(6-13 \mathrm{~mm})$, are the same size on both sides in $30 \%$ of cases, the other $30 \%$ of cases being thicker on the left. The zygomatic process (Procesus zygomaticus) may be sharp or, more frequently, with a serrated or rounded edge and with a small tubercle in almost $50 \%$ of cases.

Keywords: maxilla - anatomic landmarks

\section{Introduction}

The maxilla or the upper jaw is a pair and irregular bone, bulky, occupying the center of the face. It articulates with other bones of the face, which are arranged around it. The maxillary bones contribute to the formation of the bony palate, of the buccal and nasal cavities, of the orbit and of the infratemporal and pterygopalatine fossae. Each maxilla is formed by two bones sutured during the embryologic development: the proper maxilla and the incisive bone $[1,2,3,4]$. Maxilla has a body and four processes; the body (corpus maxillae) has the shape of a triangular pyramid, with four faces (orbital, anterior, infratemporal and nasal), four borders, a base and an apex. The anterior face (Facies anterior) or lateral is concave. At $5-8 \mathrm{~mm}$ below the orbital margin is the infraorbital foramen (Foramen infraorbitale), which may continue inferiorly as a groove. Through this opening passes the suborbital vascular-nervous bundle. The infraorbital foramen is located on the vertical line passing through the supraorbital notch of the frontal bone and the mental foramen of the mandible. Above the roots of the two premolars and 
posterior to the canine bossa on the alveolar process is located the canine fossa (Fossa canina) and anterior to the canine bossa, above the incisors, lies the myrtiform fossa $[2,5,6,7,8,9,10,11,12]$. The anterior face of the maxilla ends medially with the nasal incisure (Incisura nasalis), which along with maxilla corresponding to the opposite side, with the lower border of the nasal bones and with the intermaxillary bone defines the piriform aperture (Apertura piriformis).

\section{Materials and methods}

The morphological study of the maxilla was performed on a total of 50 skulls and on the iconography and casuistry of the Discipline of anatomy at the Faculty of Medicine, Constanta. For the external morphology of the maxilla we used the classical measurements of the anatomical landmarks; for all of these bony landmarks we studied their dimensions (length, width and depth), the shape and their location on the bone. The measurements were performed using the instruments of the anthropometric kit of the discipline of anatomy.

\section{Results and Discussions}

The canine bossa (not listed in TA) was clearly visible in $80 \%$ of cases, less prominent in $12 \%$ of cases or even absent in $8 \%$ of cases. When visible, the most commonly is oval in shape with the long axis oriented vertically (most of cases) or slightly oblique inferomedially. In $60 \%$ of cases the two fossae showed approximately equal sizes. For the rest of the $40 \%$ of the cases, the left one was larger ( 24 cases) than the right one. In $12 \%$ of cases we found a dehiscent anterior wall of the canine fossa for more than half of its height. According to $[1,2,3,4,5]$, it corresponds to the root of the canine, being placed between the canine and myrtiform fossae. Some authors $[6,7,8]$ called it canine eminence while [9] called it canine jugulum, representing a vertical bony prominence that separate the incisive and canine fossae (Figure $1)$.

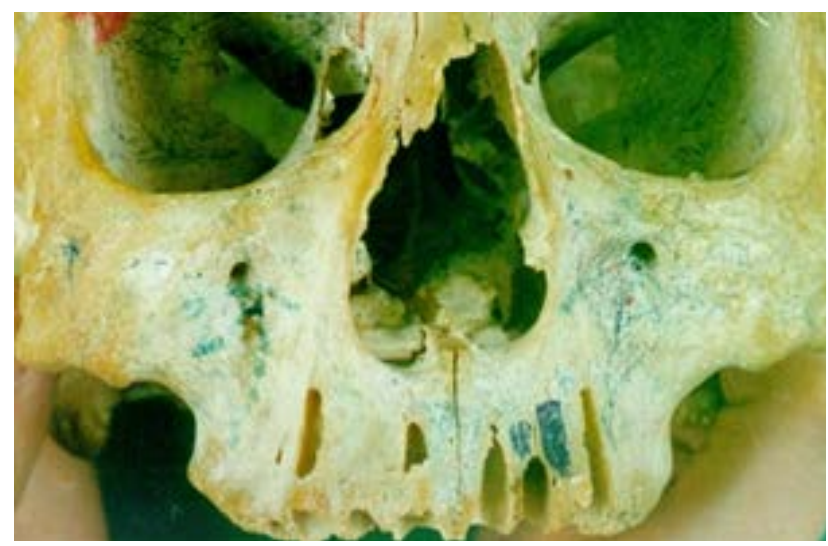

Figure 1 - Maxilla, anterior view; asymmetric piriform aperture with right inferior border located below the left one; canine bossae with thin anterior wall; right myrtiform fossa is oval, while the left one is rounded; prominent anterior nasal spine; unequal infraorbital foramens with different shapes; the right infraorbital foramen is double and located laterally to the supraorbitar notch same side, high, vertical intermaxillary suture, well visible and shallower within the inter-incisive space

The myrtiform fossa (also not mentioned in the TA) was oval, with the long axis oriented vertically and with same dimensions bilaterally in $60 \%$ of cases. In $2 / 3$ of cases was evident (deeper) on the right. In $28 \%$ of cases we found it rounded, more frequently also on the right and in $12 \%$ of cases we found it as having an irregular shape. We noticed a significant dimensional difference in relation to its shape: is larger when oval $(0.8-1.5 \mathrm{~cm}$ high and 0.5$1 \mathrm{~cm}$ transverse diameter). In about $10 \%$ of cases have it was almost flat. According to $[1,10]$ is located medially to the canine bossa, [11] homologates it as canine fossa and $[6,7,8,9]$ names it incisive fossa.

The canine fossa (Fossa canina), as the myrtiform one, has various shapes; more frequently was rounded, with an average diameter of $0.8-0.9 \mathrm{~cm}$. In $30 \%$ of cases was oval, with the vertical long axis 
of $1 \mathrm{~cm}$ in and $0.7 \mathrm{~cm}$ width. The cases of rounded canine fossa, well circumscribed and deeper, were more prevalent on the left. According to [6], the canine fossa lies superior up to the lower border of the infraorbital foramen. For [7] the canine fossa is located below the infraorbital foramen and gives attachment to the levator anguli oris muscle. Into the canine fossa is performed the trepanation of the maxillary sinus, the Caldweel-Luc procedure [7] (Figures 2 and 3).

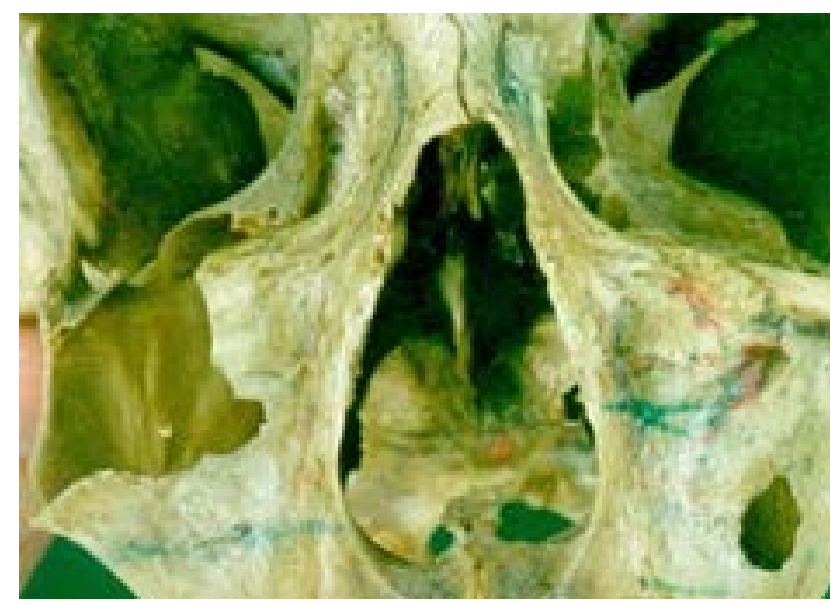

Figure 2 - Maxilla, anterior view; the anterior walls of the canine bossae are thin; symmetric piriform aperture; maxillary sinus with thin walls, with malar and orbital prominences, high, vertical intermaxillary suture, well visible within the inter-incisive space

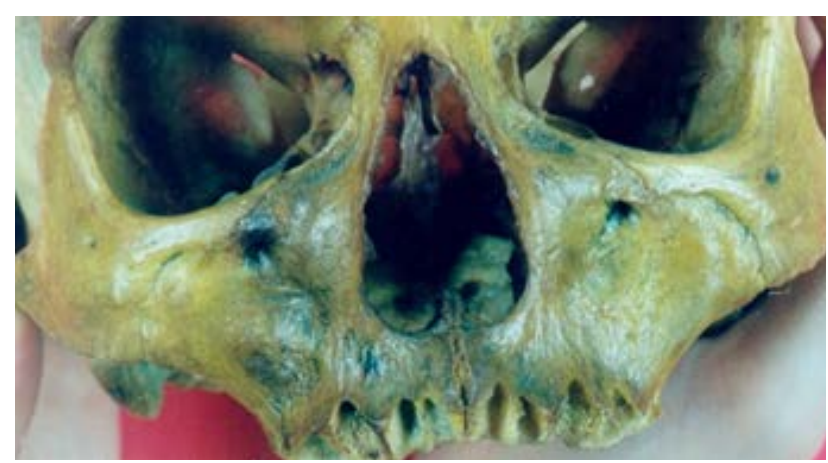

Figure 3 - Deep canine bossae, the right one more prominent; bony crest between canine and infraorbital fossae; prominent infraorbital margins; large, round infraorbital foramens; slightly asymmetric piriform aperture; bony prominence corresponding to the first premolar; vertical, short intermaxillary suture
It is well known the fact that is very difficult to determine the characteristics of the human skull, as long as the complexity and variability of the elements that constitute it impose major difficulty of this action. This aspect is more evident in the canine fossa. This depression of the maxilla is really variable in size and depth. This is often called the infraorbital fossa, but some authors state that it must distinguish between the infraorbital fossa and the canine fossa $[1,12]$, although it is not nominated in Terminologia Anatomica. Each of these terms designates a different entity. The best structure that reflects the shape of the anterolateral part of the maxilla is the infraorbital fossa. We have encountered cases in which it could make a clear distinction between the infraorbital fossa and the canine fossa, the latter being located in the infero-medial infraorbital fossa, at the bottom of the maxilla. In some cases there is an oblique inferolateral bony ridge, which separates the infraorbital fossa in a supero-lateral surface and a infero-medial one, above the canine fossa.

We established relationships between the shape of the maxilla and some morphological characteristics of the bony face: alveolar prognathism and protruding cheekbones with the depth of the infraorbital fossa. The infraorbital and the canine fossae, described by the classics are always on the anterior face of the maxilla.

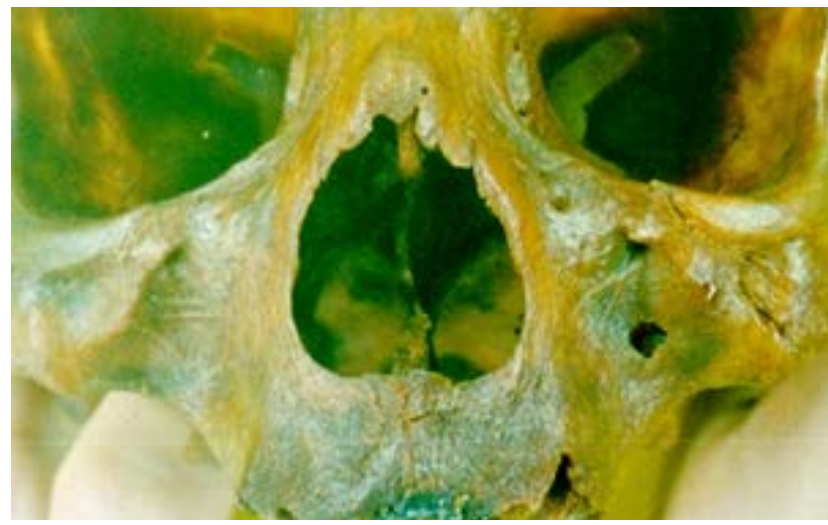

Figure 4 - Maxilla, anterior view; prominent bony crest between canine and infraorbital fossae; double left infraorbital foramen; slightly asymmetric piriform aperture

The canine crest divides the anterior face of the maxilla into an internal part, infranasal and 
an external one, infraorbital. This face is generally concave and appears as a large depression more or less profound: the infraorbital fossa (Figure 4). It is defined as infraorbital fossa the portion of the maxilla located between the inferior orbital margin superiorly, the alveolar arch inferiorly, the nasal incisure and the canine crest antero-medially and the zygomaticomalar suture and submaxillary crest postero-laterally

The canine fossa appears as a depression more or less profound and circumscribed by the infraorbital fossa. Its surface is irregular, corrugate and gives attachment to the levator anguli oris muscle. This description allows differentiating the infraorbital fossa, a constant element of the maxilla, from the canine fossa, an inconstant element (Figures 5 and 6).

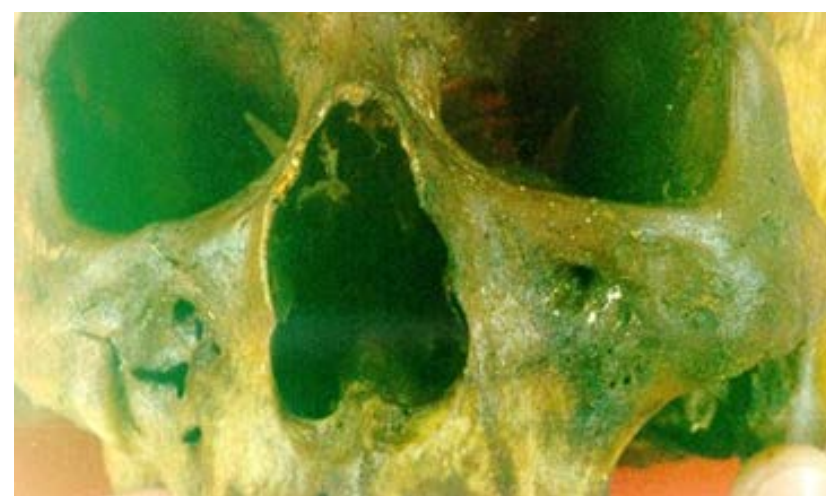

Figure 5 - Right infraorbital foramen lower than the left one; both foramens are at distance from the infraorbital margin

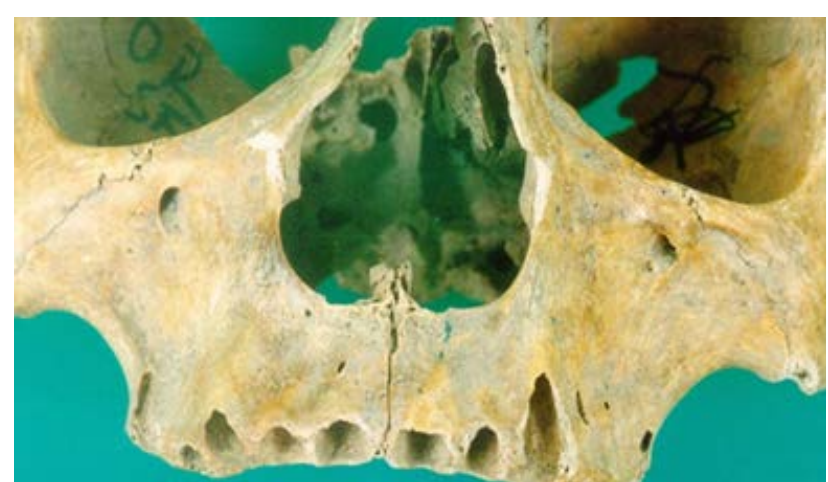

Figure 6 - Intermaxillary suture deviated to the right and slightly irregular, up to the inferior margin of the alveolar process; unequal infraorbital foramens; double left infraorbital foramen; prominent right canine bossa; shallow infraorbital and canine fossae; oval, deep myrtiform fossae
The infraorbital fossa is tightly correlated with the volume of the maxilla: on a voluminous maxilla is relatively less concave horizontally, on an average maxilla the fossa is deep and on a smaller maxilla, the infraorbital fossa is shallower.

The infraorbital foramen (Foramen infraorbitale) was circular in equal proportions (in $1 / 2$ of the cases), with a diameter of $2.5-5 \mathrm{~mm}$, or oval, with $3-5 \mathrm{~mm}$ vertical axis and $1.5-3 \mathrm{~mm}$ horizontal axis. Note the difference of shape within the same skull: circular on the left and oval on the right (most frequent case) or vice versa. It also is frequently unequal in size, usually being larger on the right. The foramen is located at a variable distance from the infraorbital margin, being lower on the left in $65 \%$ of cases. On the right side is located at 0.5 to $1.1 \mathrm{~cm}$ from the margin while on the left is at $0.6-0.9 \mathrm{~cm}$. So, a greater variability is noted on the right. Compared to the piriform aperture, the infraorbital foramen is located at 0.9 to $1.7 \mathrm{~cm}$ on the right and $1.2-1.7 \mathrm{~cm}$ on the left at. We encountered five cases of infraorbital double foramen, an aspect quoted also by $[12,13]$ (Figures 1 and 4).

The intermaxillary suture is vertical and perpendicular in only $35 \%$ of cases. In other cases it is deviated to the left in $40 \%$ of cases, and in $25 \%$ of the cases is oriented to the right. The degree of inclination is variable, making with the horizontal an angle of 95-1100.

The alveolar process (Procesus alveolaris) is wider posteriorly. There is a difference in right-to-left width, being wider on the right side in $40 \%$ of cases (6-13 mm), same size on both sides in $30 \%$ of cases and larger on the left in the other $30 \%$ of cases. The posterior opening of the alveolar border is 3.4 to $4 \mathrm{~cm}$. The most evident alveolar prominences are, in order of frequency, those of canines, incisors, premolars and molars 1 and 2 .

The zygomatic process (Procesus zygomaticus) may be sharp or, more frequently, with a serrated or rounded edge and with a small tubercle in almost $50 \%$ of cases. 


\section{Conclusions}

Considering the central position of the maxillae within the face, they would have to be perfectly symmetrical bones. This is not done, noting frequent asymmetry, particularly common in the piriform aperture but also asymmetry due to different sizes of maxillae as a whole. These asymmetries are also imposed by the different prominence, more or less exaggerated, of the anterior face of the maxillae and of the inferior orbital margin. The bony asymmetries are likely attenuated or even canceled by the arrangement of the soft tissues.

Among the anatomical landmarks that we described, some particular may be considered: the duplication of the infraorbital foramen (especially of the left maxilla) and canine fossa peculiarities related to the infraorbital fossa.

\section{Reerences}

1. Rouvière H. \& Delmas A. (1997). Anatomie Humaine déscriptive topographique et fonctionnelle. Tome 2. 14-edition. (pp. 82-87). Paris: Ed. Masson

2. Papilian V. (1998). Anatomia omului. Vol. 1. Aparatul locomotor. (pp. 40-42). Bucuresti: Ed. All

3. Cozma N. \& Frasin Gh. \& colab. (1983). Osteologie, (pp. 134-137). Iasi: Litogr. I.M.F.
4. Diaconescu N., Rottenberg N. \& Niculescu V. \& colab. (1988). Anatomia capului şi gîtului. Fascicolul I. (pp. 31-37). Timisoara: Litogr. I.M.T.

5. Bordei P., Iliescu D. \& Şapte E. (2004). Scheletul corpului uman. (pp. 131-136). Constanta: Ed. Ovidius University Press

6. Antohe D.Şt. \& Varlam H. (2004). Sistemul locomotor. Scheletul. (pp. 156-166). Iasi: Ed. Junimea

7. Panaitescu V., Gănuță N. \& Roșu M. (2002). (pp. 110-118). Bucuresti: Ed. Med. Nationala

8. Gray's Anatomy. (2005). Anatomy. The Anatomical Basis of Cilinical Practice. (pp. 477-480). Edinburgh: Ed. Elsevier Churchil Livingstone

9. Kamina P. (2002). Précis d'Anatomie Clinique. Tome 2. (pp. 57-60). Paris: Ed. Maloine

10. Bouchet A. \& Cuilleret J. (1991). Anatomie topographique, descriptive et fonctionnelle. Vol.1. Le système nerveux central, la face, la tête etles organes des sens. (pp. 331-336). Paris: Ed. Simep.

11. Tardif B. \& Chevrel J.P. (1996). Les os de la face. In: Anatomie clinique. Tête et cou. (pp. 37-42). Paris: Ed. Springer-Verlag

12. Platzer W. (2001). Anatomie 1. Appareil Locomoteur. (pp. 296-299). Paris: Ed. Flammarion Médecin-Scieces

13. Scarfe W.C. et al. (1998). Panoramic radiographic patterns of the infraorbital canal and anterior superior dental plexus. Dentomaxillofac. Radiol. $27(2), 85-92$

14. ******* Terminologia Anatomica. International Anatomical Terminology (pp. 14-15). Stuttgart: Ed. Thieme-Verlag 